\title{
A Rare Case of Epidermolysis Bullosa With Sudden Airway Obstruction
}

\author{
Amal Faisal Al Abdullaa, b, Mohamed Hassan Bella ${ }^{\text {a }}$, Shilpa Prabhu ${ }^{\mathrm{a}}$
}

\begin{abstract}
Epidermolysis bullosa (EB) is a rare condition which may present at birth or early infancy. These patients have a poor prognosis with high mortality rate. Tracheolaryngeal stenosis or stricture is the most significant ENT complication which may result in death from airway obstruction. We present a case of EB presenting with sudden onset airway obstruction, requiring immediate airway management. The epidemiology, pathogenesis and clinical presentation of this disease are discussed as well as the diagnosis and management. ENT surgeons as well as other health care providers must be aware of the potential risks to the airway in these patients.
\end{abstract}

Keywords: Epidermolysis bullosa; Supraglottic; Subglottic; Stenosis; Skin; Lesions; Airway; Obstruction

\section{Introduction}

Epidermolysis bullosa (EB) is a group of inherited connective tissue diseases, characterized by multiple blisters which affect the skin and mucosal membranes in response to minor or no obvious mechanical trauma [1].

This is a chronic disease with a poor prognosis. Mortality is due to skin complications which include squamous cell carcinoma with resultant metastases, renal failure or chronic mechanical obstruction [2].

We present a case of EB presenting with sudden onset airway obstruction, requiring immediate airway management. The epidemiology, pathogenesis and clinical presentation of this disease are discussed as well as the diagnosis and management.

\section{Case Report}

The patient was a 3-year-old male, of Jordanian origin. He was a known case of EB and he presented to the emergency depart-

Manuscript accepted for publication February 02, 2016

aENT Department, BDF Hospital, Kingdom of Bahrain

bCorresponding Author: Amal Al Abdulla, Bahrain Defence Force Hospital, PO Box 28347, Riffa, Kingdom of Bahrain. Email: amalffa4@hotmail.com

doi: http://dx.doi.org/10.14740/ijcp234w ment with a history of noisy breathing which started suddenly and progressed over a period of 3 days prior to this visit.

The patient had a history of recurrent episodes of breathing difficulties and noisy breathing for the past $1-2$ years. On several occasions, the patient required hospital admission during which he was treated with ipratropium bromide/albuterol (Combivent) nebulization and steroid containing medications.

He also gave a history of skin lesions on multiple areas of the body which were initially noticed by the family at the age of 8 months. These lesions appeared secondary to minor trauma or friction and occasionally there was no prior injury (Fig. 1). They initially appeared as small fluid filled lesions which increased in size. They then burst leaving a moist erythematous surface. Healing would only occur with the aid of a combination of beta-sitosterol and an antibiotic ointment such as bacitracin, neomycin and fucidin. These medications were prescribed by either a general practitioner or a dermatologist.

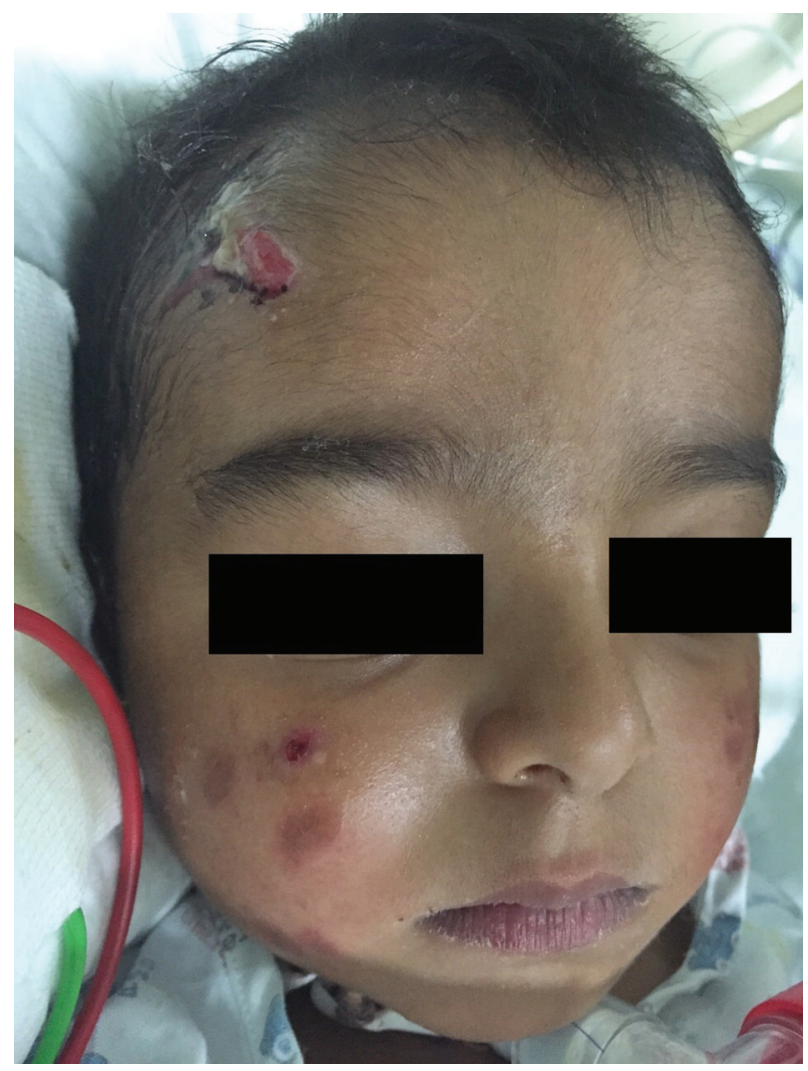

Figure 1. Multiple blisters seen on the patient's cheeks and forehead. 


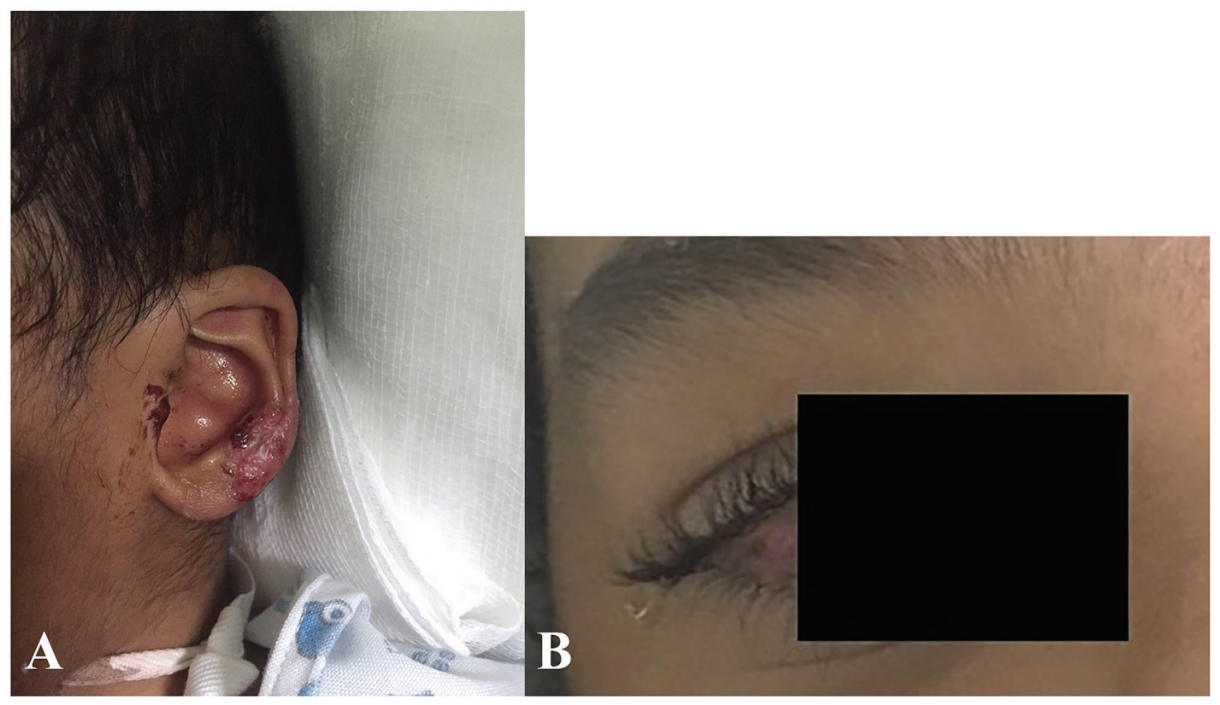

Figure 2. (A) Skin blister involving the patient's auricle.

(B) Corneal erosions seen in the same patient with EB.

The nails were brittle and broke frequently. In addition, he had lesions involving the eyes which also started at the age of 8 months. He required frequent visits to the ophthalmologist and had undergone four eye surgeries in the past.

The parents are second-degree cousins from the mother's side. Both parents were healthy with no history of similar illnesses. Parents had four children: three girls and one boy. The patient is the third in order among them. His youngest sister also diagnosed with EB had passed away at the age of 2 years due to sudden airway obstruction. The other two older sisters were healthy with no history of any medical illnesses.

On examination, the child had multiple skin lesions involving multiple areas of the body including the face, ears, back, limbs, fingers and eyes (Fig. 2A, B). The nails were brittle and lesions were found in the nail bed.

Vital signs were stable; however, he had signs of respiratory distress. This included intercostal and retrosternal retraction on inspiration and audible biphasic stridor.

Laryngoscopy was done using a flexible endoscope which showed a supraglottic lesion, measuring $1 \mathrm{~cm}$ in diameter and causing significant airway obstruction (Fig. 3).

Tracheostomy was done due to difficult laryngeal intubation and the child underwent upper rigid endoscopic examination under general anesthesia. This showed a supraglottic lesion measuring approximately $1 \mathrm{~cm}$ in diameter, red in color and firm in consistency. This lesion was excised and sent for histopathologic analysis. Further examination of the larynx showed significant subglottic stenosis with a slit like subglottic space. Endoscopy of the bronchial tree was done using a flexible scope through the tracheostomy tube which showed no lesions or narrowing in the trachea, carina or bronchial tree down to second generation branches.

Two punch skin biopsies were taken, one specimen was sent for histopathology and the second for immunohistochemistry.

The postoperative period was uneventful and the patient was kept in the ward for a few days for tracheostomy care. Swabs were taken from the tracheal secretions and sent for culture and sensitivity which showed growth of moraxella catarrhalis and methicillin-resistant Staphylococcus aureus (MRSA) and the patient was started on antibiotic treatment according to bacterial sensitivity. The patient was sent home on day 7 with the tracheostomy tube in place and the parents were advised regarding the home care of a tracheostomized patient.

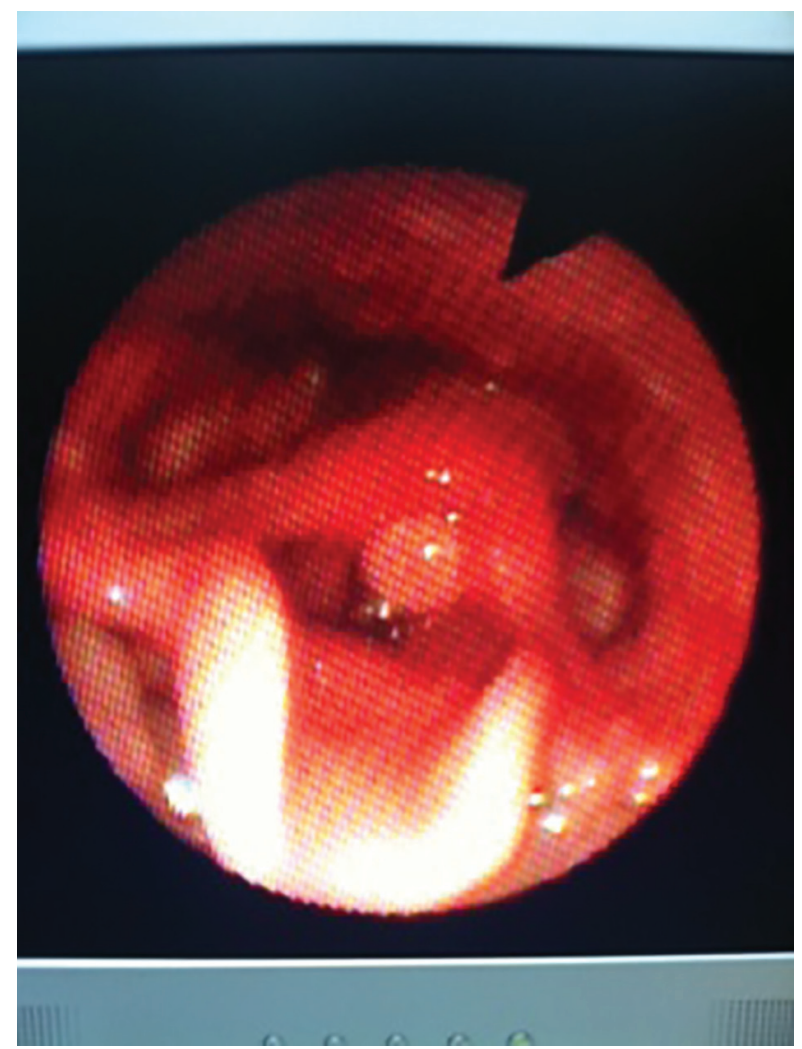

Figure 3. Rigid laryngoscopy under general anesthesia, showing a supraglottic lesion causing significant airway compromise. 


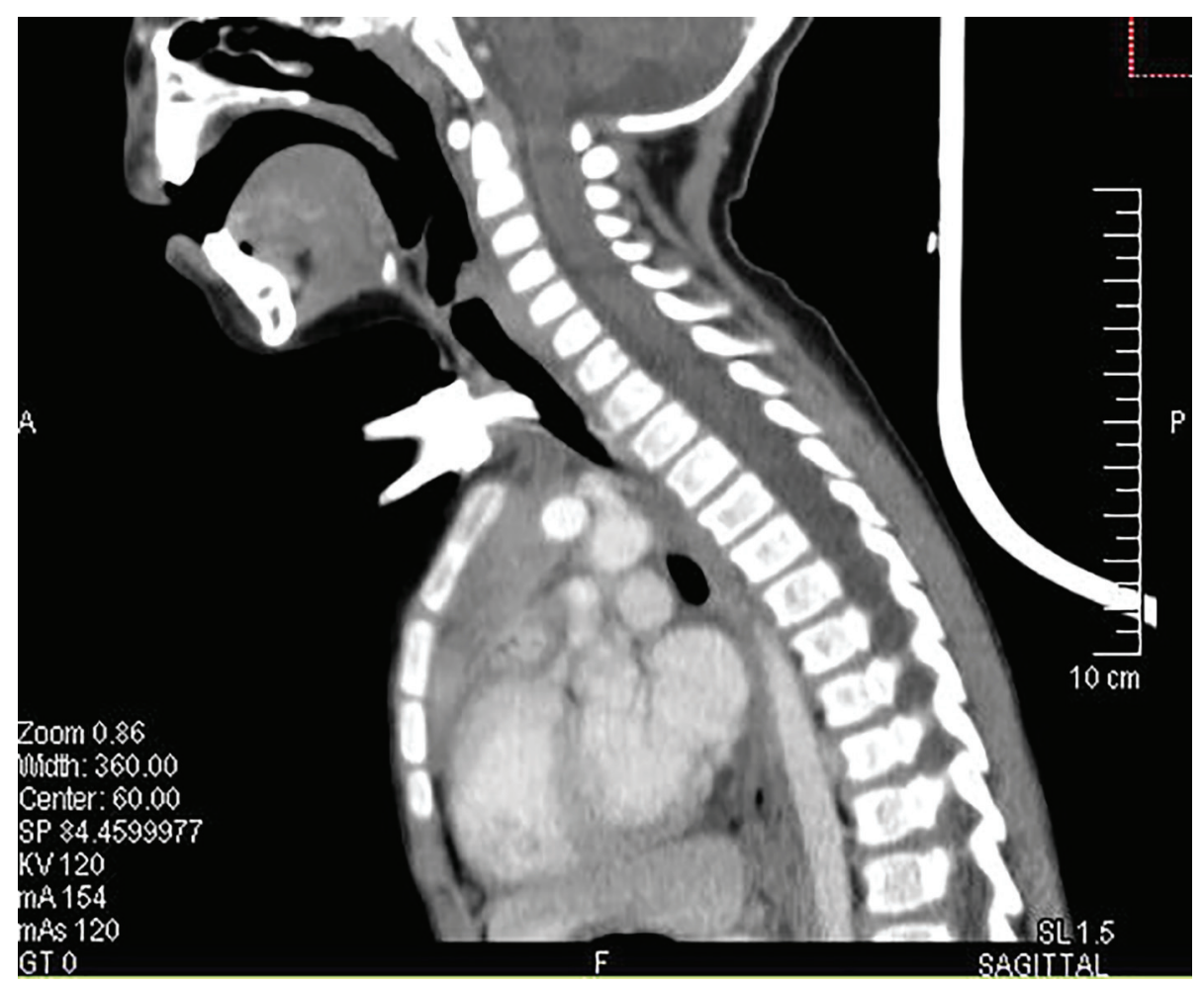

Figure 4. CT scan of the neck showing significant subglottic stenosis.

CT scan of the neck showed subglottic narrowing (Fig. 4).

Histopathology report of the biopsied laryngeal tissue showed remarkable neutrophilic infiltration in the underlying stroma along with granulation tissue formation.

Skin biopsy studied at multiple levels revealed hyperkeratosis, epidermal hyperplasia with hypergranulosis. The upper and deep dermis showed perivascular lymphoplasmacytic infiltrate and focal subepidermal clefting.

Direct immunofluorescence studies showed IgG deposition at the dermal-epidermal junction while negative for IgA, $\mathrm{C} 3$ and $\mathrm{C} 1 \mathrm{q}$.

\section{Discussion}

EB is a rare condition [3], which was first described in 1981 [4]. Studies have shown that EB in consanguineous families from different parts of the Middle East was found to have distinctive genetic mutations not found in US and European EB patients [5]. $[2,6]$.

EB may present at any stage from birth to 2 years of age

The incidence is equal in both males and females [7]. Our patient as well as his sister both had the same disease which presented in infancy. Our patient first started to develop skin lesions at the age of 8 months, when the parents noticed skin blistering and injury which was caused by either minor injury or friction. These lesions involved different parts of the skin including the face, ears, back, limbs, fingers and nails. In addition, these scars failed to heal spontaneously and required medical therapy in the form of skin ointments prescribed by the dermatologist. The disease has been classified into four major types, epidermolysis bullosa simplex (EBS), dystrophic epidermolysis bullosa (DEB), junctional epidermolysis bullosa (JEB) and Kindler. This classification was based on the ultrastructural level of the skin within which the blisters develop, either in response to trauma form minor friction or spontaneously [8].

In our case, skin biopsy showed hyperkeratosis, epidermal hyperplasia with hypergranulosis. The upper and deep dermis shows perivascular lymphoplasmacytic infiltrate and focal subepidermal clefting.

More than 1,000 genetic mutations have been identified in $\mathrm{EB}$ [2].

EB is characterized by blister lesions involving the skin and mucous membranes. These blisters most commonly appear at sites of friction and minor trauma such as the feet and hands but may occur in any other part of the body including internal organs, such as the esophagus, stomach and respiratory tract, without any apparent friction $[2,8]$. Extracutaneous manifestations include anemia, growth retardation, caries, enamel hypoplasia, gastrointestinal complications including pyloric stenosis, ocular erosion, pseudodactyly, as well as respiratory tract complications including laryngotracheal stenosis or stricture $[2,8]$.

Similarly our patient had several lesions involving the skin as well as recurrent ocular erosions and airway obstruction due to laryngeal stenosis and supraglottic lesion.

These patients have a poor prognosis with high mortality rate. Death may result from renal failure, chronic mechanical obstruction which may be respiratory or gastrointestinal. Mortality may also result from skin complications, sepsis and 
squamous cell carcinoma with $80 \%$ metastases and death [2, 8]. Tracheolaryngeal stenosis or stricture is the most significant ENT complication which may result in death from airway obstruction $[9,10]$. This was found in our case, he presented to the emergency department due to acute airway obstruction requiring prompt management. He had a difficult endotracheal intubation due to an obstructing supraglottic lesion and significant narrowing of the laryngeal inlet. We also take into consideration that his youngest sister also diagnosed with EB had died at the age of 2 years due to sudden airway obstruction. Based on these findings, it was decided to perform a tracheostomy as a life saving measure. The supraglottic lesion was excised and sent for histopathologic analysis.

Other uncommon complications included chronic otitis media, chronic otitis externa, and hearing loss [9]. Our patient had normal ear examination and normal hearing. In one literature review of reports of laryngeal EB over a 20-year period, only 18 cases of laryngotracheal EB have been documented and most of these were diagnosed with the JEB subtype [11]. In a literature review that we have conducted using a Medline search, we found only nine cases of laryngeal EB have been documented over the past 14 years. By age 6 and 9, the cumulative risk of respiratory complications is $39.8 \%$ in Herlitz subtype and $12.8 \%$ in non-Herlitz subtype of JEB [8, 9].

JEB is a generalized severe form of EB and has an extremely poor prognosis, with a mean age of death at 5 months old and the majority die before the age of 3 years [12]. Airway obstruction may present suddenly and as an acute emergency requiring immediate intervention. Specifically in JEB subtype and two rare subtypes of generalized EBS [9]. Our patient presented at the age of 3 years with sudden airway obstruction and his sister passed away at the age of 2 years due to sudden airway obstruction. In view of this poor prognosis of patients with EB, close observation is necessary due to the potential risk of airway compromise. Elective tracheostomy should be considered in these cases to reduce the risk of death from sudden airway obstruction.

\section{Conclusion}

EB is a life threatening disease with a potentially high risk of death from sudden airway compromise. ENT surgeons as well as other health care providers must be aware of the potential risks to the airway in these patients. Airway management including an elective tracheostomy must be considered in EB patients specifically in the high risk subtypes such as JEB.

\section{Conflicts of Interest}

I have no potential conflicts of interest.

\section{References}

1. Murat-Susic S, Husar K, Marinovic B, Skerlev M, Buzina DS, Kljenak A, Martinac I. [Skin care and wound management in patients with inherited bullous epidermolysis]. Acta Med Croatica. 2014;68(Suppl 1):31-38.

2. Surasak Puvabanditsin, Erik Brandsma, Nisha Patel, et al. Pediatric Epidermolysis Bullosa. Medscape. Updated: 7, 2013.

3. Goldschneider KR, Good J, Harrop E, Liossi C, LynchJordan A, Martinez AE, Maxwell LG, et al. Pain care for patients with epidermolysis bullosa: best care practice guidelines. BMC Med. 2014;12:178.

4. Koebner H. Hereditare anlage zur blasenbildung (epidermolysis bullosa hereditaria). Dtsch Med Wochenschr. 1886;12:21-22.

5. Abu Sa'd J, Indelman M, Pfendner E, Falik-Zaccai TC, Mizrachi-Koren M, Shalev S, Ben Amitai D, et al. Molecular epidemiology of hereditary epidermolysis bullosa in a Middle Eastern population. J Invest Dermatol. 2006;126(4):777-781.

6. Hon KL, Li JJ, Cheng BL, Luk DC, Murrell DF, Choi PC, Leung AK. Age and etiology of childhood epidermolysis bullosa mortality. J Dermatolog Treat. 2015;26(2):178182.

7. Pfendner E, Uitto J, Fine JD. Epidermolysis bullosa carrier frequencies in the US population. J Invest Dermatol. 2001;116(3):483-484.

8. Fine JD, Bruckner-Tuderman L, Eady RA, Bauer EA, Bauer JW, Has C, Heagerty A, et al. Inherited epidermolysis bullosa: updated recommendations on diagnosis and classification. J Am Acad Dermatol. 2014;70(6):11031126.

9. Fine JD, Eady RA, Bauer EA, Bauer JW, Bruckner-Tuderman L, Heagerty A, Hintner H, et al. The classification of inherited epidermolysis bullosa (EB): Report of the Third International Consensus Meeting on Diagnosis and Classification of EB. J Am Acad Dermatol. 2008;58(6):931950.

10. Thompson JW, Ahmed AR, Dudley JP. Epidermolysis bullosa dystrophica of the larynx and trachea. Acute airway obstruction. Ann Otol Rhinol Laryngol. 1980;89(5 Pt 1):428-429.

11. Liu RM, Papsin BC, de Jong AL. Epidermolysis bullosa of the head and neck: a case report of laryngotracheal involvement and 10-year review of cases at the Hospital for Sick Children. J Otolaryngol. 1999;28(2):76-82.

12. Yang CS, Kroshinksy D, Cummings BM. Neonatal junctional epidermolysis bullosa: treatment conundrums and ethical decision making. Am J Clin Dermatol. 2014;15(5):445-450. 